\title{
Potential role of WSB1 isoforms in growth and survival of neuroblastoma cells
}

\author{
Keren Shichrur ${ }^{1,2}$, Galina Feinberg-Gorenshtein ${ }^{1,2}$, Drorit Luria ${ }^{3}$, Shifra Ash ${ }^{3}$, Isaac Yaniv ${ }^{1,2,3}$ and Smadar Avigad ${ }^{1,2,3}$
}

\begin{abstract}
BACKGROUND:WD repeat and SOCS box containing protein 1 (WSB1) generates three isoforms that were found to play a role in cancer cell growth and tumor progression. We have studied their expression in neuroblastoma (NB).

METHODS: The behavior of the expression levels of the WSB1 isoforms was analyzed in NB cell lines, in an in vivo NB xenograft mouse model, and in primary NB tumors using real-time PCR. Effective WSB1 small interfering RNAs were transfected into cultured NB cell lines, and cell viability was analyzed using XTT assay and flow cytometry.
\end{abstract}

RESULTS: A significant predominance of the WSB1 isoform 3 $\left(\mathrm{WSB}^{3}\right)$ expression level was demonstrated in all NB systems examined. Correspondingly, combination of WSB1 ${ }^{3}$ silencing together with WSB1 isoforms 1+2 silencing in NB cells showed reduced growth, enhanced apoptosis rate, and increased sensitivity to chemotherapeutic agents, specifically related to low expression of $\mathrm{WSB}^{3}{ }^{3}$

CONCLUSION: Our results point to a possible differential role of WSB1 isoforms in NB and suggest WSB1 ${ }^{3}$ as a target for therapy in NB.

$\mathbf{N}$ euroblastoma (NB) is one of the most common pediatric solid tumors in children aged $\leq 5$ years and accounts for $15 \%$ of all pediatric cancer deaths (1). NB tumors exhibit a remarkable heterogeneity with respect to clinical behavior, ranging from spontaneous regression or differentiation with favorable outcome to a rapid progression with poor outcome, despite multimodal therapy (2-5). This extreme clinical heterogeneity reflects the complexity of the events associated with development and progression of the disease. Analyzing the changes in gene expression at diagnosis and during NB tumor progression could lead to the understanding of the molecular characteristics of NB tumor initiation and progression processes and, eventually, to improvement in therapy. NB tumor progression is accompanied by changes in gene expression affecting angiogenesis, invasion, proliferation, and apoptosis, as well as hypoxia and resistance to chemotherapeutic agents (6-9). The identification of genes involved in these processes could lead to a significant improvement in understanding the molecular mechanisms of NB tumor development, and inhibition of their expression might be a new way of targeting NB. WD repeat and SOCS box containing protein 1 (WSB1) was described before as a potential stress-induced gene that supports pancreatic tumor progression by modulating the alternative splicing of three isoforms after exposure to several stress signals (10). Moreover, Chen et al. (11) had already identified WSB1 to have a gene dosage effect and reported that its high expression is associated with better survival in NB patients. Our main purpose in this study was to examine the behavior of the expression levels of the WSB1 isoforms in several NB cell systems and to understand the role of WSB1 isoforms in relation to $\mathrm{NB}$ tumors using small interfering RNA (siRNA) technology. In this article, we describe a significant predominance of specific WSB1 isoform in all NB cell systems examined and its role in tumor cell growth. We suggest that modulation of WSB1 isoforms might be a potential option for therapy in NB.

\section{RESULTS \\ WSB1 Expression in NB Cell Lines, Tumor Xenografts, and Primary NB Tumors}

The WSB1 gene generates, by alternative splicing, three variant transcripts encoding distinct protein isoforms. We developed a specific primer assay to analyze independently the expression of WSB1 isoforms $1+2\left(\mathrm{WSB}^{1+2}\right)$ and isoform $3\left(\mathrm{WSB}^{3}\right)$ by real-time quantitative PCR (RQ-PCR). First, the expression levels of WSB1 isoforms were measured in four human NB cell lines (SK-N-SH, SH-SY5Y, IMR32, and SH-EP). As shown in Figure 1a, the relative expression level of $\mathrm{WSB}^{3}$ was two- to fivefold higher than the expression of WSB $1^{1+2}$ in all cell lines studied. In order to measure the expression of WSB1 isoforms in vivo, we developed an NB mouse xenograft model. Injection of $10^{7} \mathrm{MNA}$ (MYCN gene amplified) Nub6 cells subcutaneously resulted in the establishment of tumors in $100 \%$ of 6 - to 8 -week-old nonobese diabetic/severe combined immunodeficiency (NOD/SCID) mice within a few days after injection. Two weeks after injection, all 34 mice showed generation of a tumor. RNA was extracted from the tumors, and the expression levels of WSB1 isoforms were measured using our RQ-PCR-specific primer assay. The relative expression level of the $\mathrm{WSB}^{3}{ }^{3}$ isoform

${ }^{1}$ Molecular Oncology, Felsenstein Medical Research Center, Petah Tikva, Israel; ${ }^{2}$ Sackler Faculty of Medicine, Tel Aviv University, Tel Aviv, Israel; ${ }^{3}$ Pediatric Hematology Oncology Center, Schneider Children's Medical Center of Israel, Petah Tikva, Israel. Correspondence: Smadar Avigad (smadar_avigad@clalit.org.il)

Received 10 October 2012; accepted 1 October 2013; advance online publication 12 February 2014. doi:10.1038/pr.2014.2 
in the xenograft tumor cells was 4.7 -fold higher than the expression of $\mathrm{WSB}^{1+2}$ (Figure $1 \mathbf{b}$ ). Interestingly, the same cell line grown in vitro showed only 2.1 -fold increase, suggesting a possible role of $\mathrm{WSB} 1^{3} / \mathrm{WSB}^{1+2}$ ratio in tumor cell development.

Finally, we analyzed the expression pattern of $\mathrm{WSB}^{1+2}$ and $\mathrm{WSB}^{3}$ isoforms in six primary NB MNA tumor samples. Average expression level of $\mathrm{WSB}^{3}$ isoform was again 26 -fold higher than $\mathrm{WSB}^{1+2}$ expression level, indicating the predominance of the $\mathrm{WSB}^{3}$ isoform also in primary NB samples (Figure 1b).

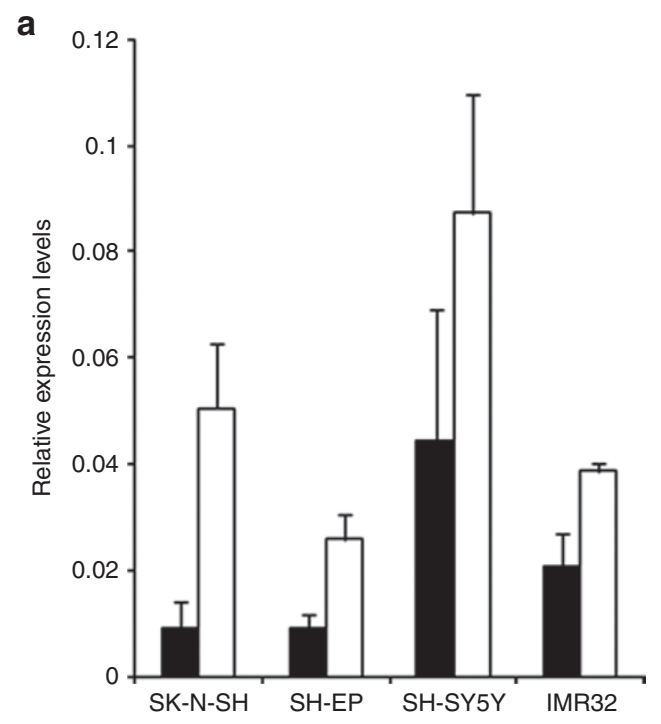

b

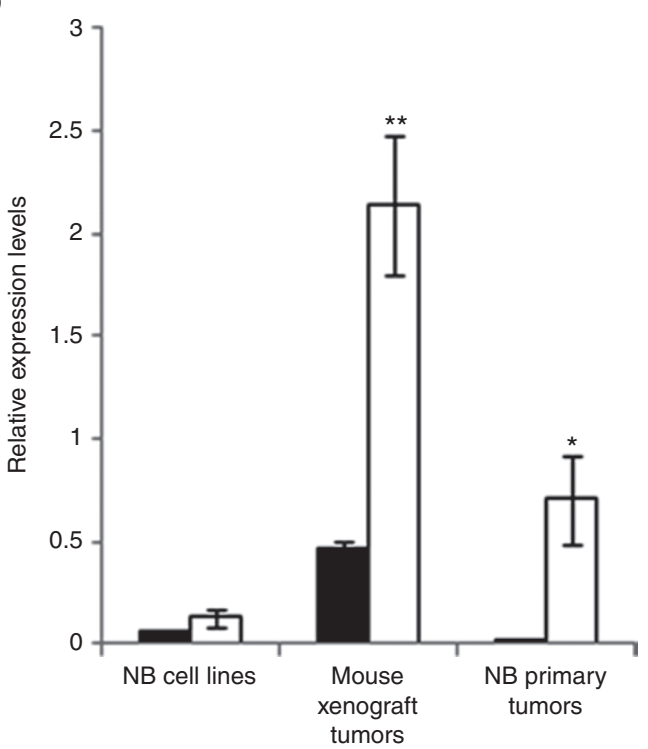

Figure 1. WD repeat and SOCS box containing protein 1 isoform 3 $\left(\mathrm{WSB} 1^{3}\right)$ is highly expressed in mice neuroblastoma (NB) xenografts and human NB cell lines. (a) Relative expression of WSB $1^{1+2}$ (black bars) and WSB ${ }^{3}$ (white bars) mRNAs was assessed by real-time quantitative PCR (RQ-PCR) in four NB cell lines (SK-N-SH, SH-EP, SH-SY5Y, and IMR32). (b) Average expression of WSB $1^{1+2}$ (black bars) and WSB1 ${ }^{3}$ (white bars) mRNAs was assessed by RQ-PCR of 5 NB cell lines (SK-N-SH, SH-EP, SH-SY5Y, IMR32, and Nub6), 34 xenografted tumors, and 6 primary NB MNA tumor samples. Values are expressed as the mean $\pm \mathrm{SE}\left({ }^{*} P<0.05 ;{ }^{* *} P<0.0001\right)$.

\section{WSB1 Enhances Cell Proliferation}

The function of WSB1, particularly of the WSB13 $1^{3}$ isoform, in human cancer is mostly unknown. We investigated the role of WSB1 isoforms in cell viability and apoptosis by XTT assay and flow cytometry (fluorescence-activated cell sorting analysis). We inhibited WSB1 expression by transfecting SK-N-SH and SH-EP NB cell lines with several siRNAs that target the three isoforms. Twenty four to $72 \mathrm{~h}$ following transfection, siRNA1 efficiently caused a reduction of $90-95 \%$ of $\mathrm{WSB}^{1+2}$ expression levels and a $60-70 \%$ reduction of $\mathrm{WSB}^{3}$, whereas siRNA2 reduced mostly $\mathrm{WSB}^{1+2}$ expression (90-95\% of $\mathrm{WSB}^{1+2}$ and only $10-20 \%$ of $\mathrm{WSB}^{3}$; Figure 2 ). We were not able to target the $\mathrm{WSB}^{3}$ isoform specifically by siRNAs in a sufficient manner. Our assay enabled us to study the effect of WSB1 ${ }^{3}$ downregulation using two silenced cell lines, each representing different expression levels of the $\mathrm{WSB}^{3}$ isoform.

To assess the effect of WSB1 isoform reduction on cell viability, the siRNA1- and siRNA2-silenced cell lines were analyzed using XTT viability assay and compared with untransfected cell line. Results indicate that cells transfected with WSB1 siRNA1 are less viable than control cells or cells transfected with siRNA2. Forty-eight hours after transfection, only $45 \%$ of the cells were viable in the siRNA1-transfected cells (Figure 3a). Apoptosis in the siRNA1-transfected cells was two- to threefold higher than that in siRNA2-transfected and control cells, indicating that the levels of $\mathrm{WSB}^{3}$ are responsible for this effect (Figure 3b). In fact, siRNA2-silenced cells displayed similar characteristics when compared with the control cells, suggesting that WSB1 ${ }^{1+2}$ silencing alone had no effect on cell viability. Similar results were obtained with SK-N-SH cells (data not shown). Taken together, these results strongly

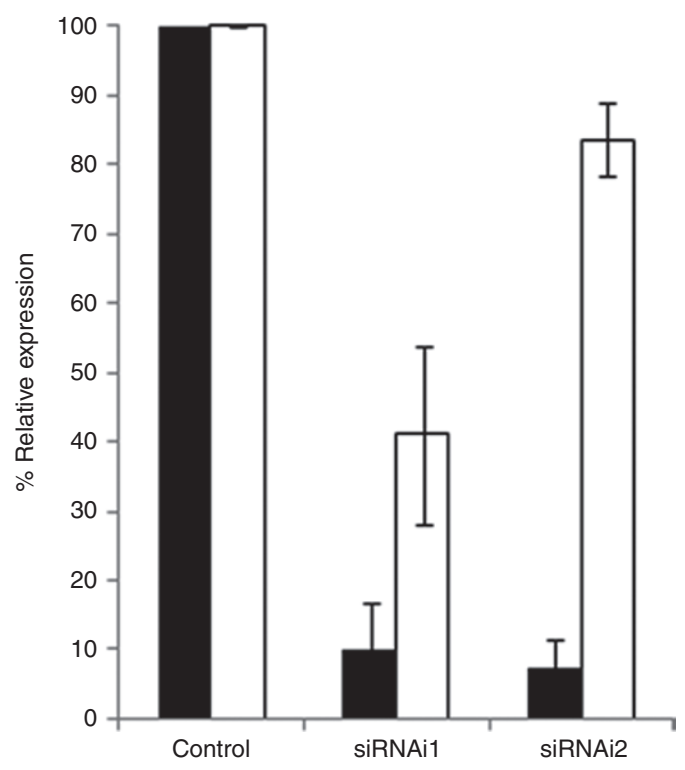

Figure 2. WD repeat and SOCS box-containing protein 1 (WSB1) downregulation in neuroblastoma (NB)-silenced cell line. Expression of WSB $1^{1+2}$ (black bars) and WSB $1^{3}$ (white bars) mRNAs is shown in SH-EP cells transfected with small interfering RNA (siRNA) 1 and siRNA2 directed against WSB1. Values are expressed as the mean \pm SE of results performed in duplicates. 


\section{Articles $\mid$ Shichrur et al.}
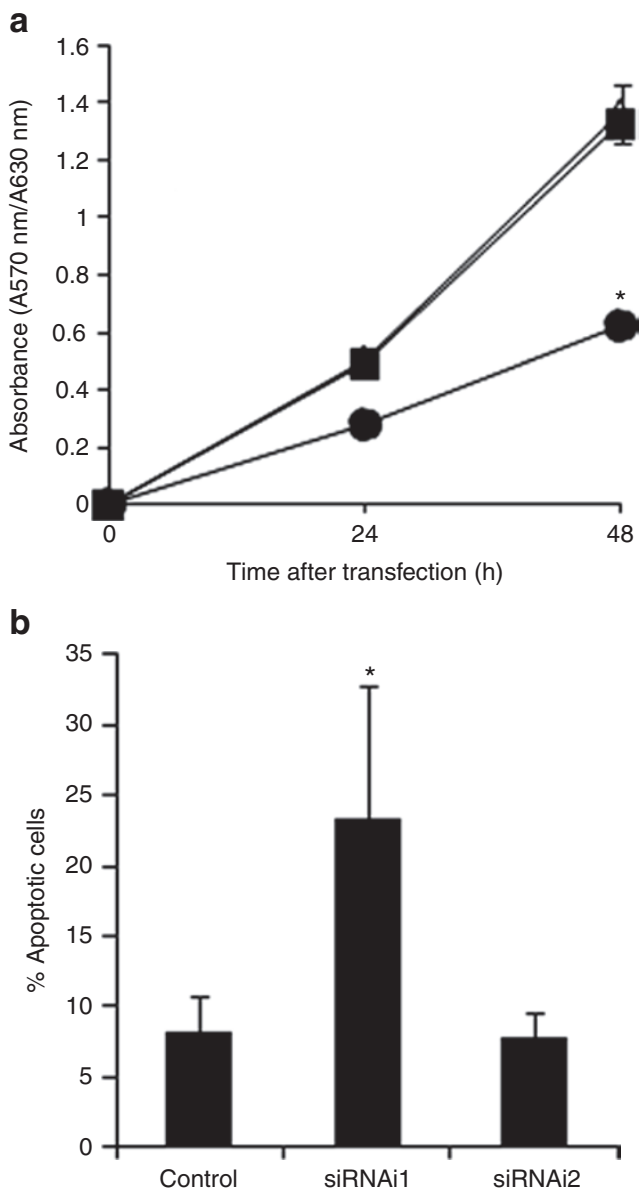

Figure 3. WD repeat and SOCS box containing protein 1 (WSB1) downregulation decreases cell growth. (a) Cell viability measured by XTT assay in WSB1 siRNA1-transfected SH-EP cells (black circles), siRNA2-transfected SH-EP cells (black squares), and untransfected SH-EP cells (white triangle) 24 and $48 \mathrm{~h}$ after transfection. (b) SH-EP cells transfected with the WSB1 small interfering RNA and stained with propidium iodide $24 \mathrm{~h}$ after transfection. Apoptosis was measured by flow cytometry (fluorescenceactivated cell sorting). Values are expressed as the mean $\pm \mathrm{SE}$ of combined results from three independent experiments $\left({ }^{*} P<0.05\right)$.

suggest that downregulation of WSB $1^{3}$ decreases cell growth by enhancing cell death.

\section{WSB $1^{3}$ Reduces Susceptibility to Stress-Induced Cell Death}

Because expression of WSB1 was found to be affected by stressinduced cell death by chemotherapeutic agents (10), we investigated whether it could affect the susceptibility of NB cells to chemotherapeutic agents. We subjected SH-EP-silenced cells to treatment with 100 or $150 \mu \mathrm{mol} / \mathrm{l}$ doxorubicin, known to induce apoptosis, and measured cell viability after $24 \mathrm{~h}$ by XTT assay. Cells transfected with siRNA1 were more sensitive to doxorubicin in a dose-dependent manner than siRNA2-transfected cells and control cells (Figure 4a). The assay was also performed following treatment with 20 and $30 \mu \mathrm{mol} / \mathrm{l}$ cisplatin for $48 \mathrm{~h}$. A profile similar to that of doxorubicin-treated cells was obtained; cells transfected with siRNA1 were more sensitive to the drug than siRNA2-transfected cells and control cells (Figure $4 \mathrm{~b}$ ). These findings imply that downregulation a
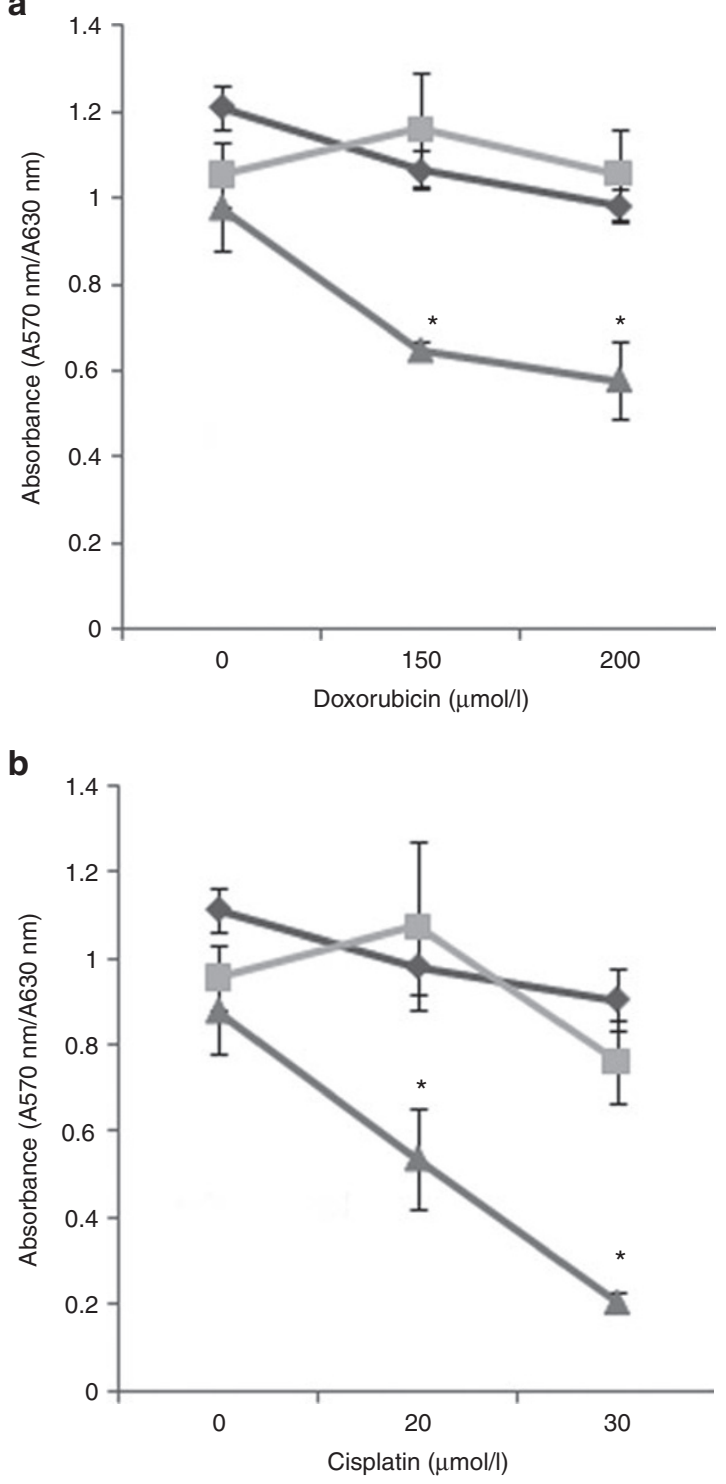

Figure 4. WD repeat and SOCS box containing protein 1 isoform 3 (WSB $1^{3}$ ) downregulation increases cell sensitivity to chemotherapeutic agents. SH-EP cells transfected with small interfering RNA (siRNA) 1 (gray triangle) and siRNA2 (gray squares) and untransfected SH-EP cells (gray diamonds) were evaluated by XTT assay without and after treatment with two concentrations of (a) doxorubicin or (b) cisplatin. Values are expressed as the mean \pm SD of combined results from two independent experiments performed in triplicates $\left({ }^{*} P<0.05\right)$.

of $\mathrm{WSB} 1^{3}$ results in increased apoptosis and decreased resistance to chemotherapy treatment and further suggest a role for $\mathrm{WSB} 1^{3}$ isoform in apoptosis.

\section{DISCUSSION}

Alternative splicing is a significant process that allows production of discrete isoforms with distinct functions through spatial and temporal regulation (12). It had been already demonstrated that isoforms of WSB1 contribute to the proliferation of pancreatic cancer cells, as well as to chemotherapy resistance (10). In this study, we analyzed the behavior of the expression of the three WSB1 isoforms in NB. We analyzed the expression levels 
of the WSB1 isoforms in NB cell lines, in an in vivo xenograft mouse model, and in primary NB MNA tumors. We identified a significant predominance of the WSB $1^{3}$ isoform in all NB cell lines tested, as well as in the in vivo xenograft mouse model. This predominance was also shown in six primary NB MNA tumors. The fact that it behaves in the same way in all systems examined makes WSB1 $1^{3}$ isoform an interesting candidate for research in NB. WSB1 was also found to be a prognostic factor in NB (11). Chen et al. (11) showed that an increased copy number correlated with its overexpression and better survival rate in NB. In their study, WSB1 isoforms were not analyzed, so the contribution of each isoform to tumor progression was unknown. Although the role of the WSB1 $1^{3} / \mathrm{WSB} 1^{1+2}$ ratio in NB was not defined through our approach, the interesting fact that the $\mathrm{WSB} 1^{3} / \mathrm{WSB} 1^{1+2}$ ratio was increased in the 2 -week xenograft mouse tumors compared with that in the same cell line grown in vitro may imply a possible role of these isoforms in NB tumors. A similar modulation by alternative splicing of the expression of WSB1 isoforms was already observed in pancreatic nude mice xenografts and in human pancreatic tumors (10). The similarity in the behavior of WSB1 isoforms in the two types of tumors suggests the possibility of a more general role of these isoforms in tumor development. WSB1 is a SOCSbox-containing WD40 protein belonging to a diverse class of E3 ubiquitin ligase that has specificity and recognizes the target protein for degradation through the ubiquitin process $(13,14)$. Recent studies showed that thyroid-hormone-activating type 2-iodothyronine deiodinase (D2) and homeodomain-interaction protein kinase 2 (HIPK2) are WSB1 substrates $(13,15,16)$. Although the function of WSB1 is mostly unknown, its structural domains imply a possible role in degrading oncogenes through the ubiquitin process, thus limiting tumor growth (15). The molecular mechanism associating $\mathrm{WSB}^{3}$ to $\mathrm{NB}$ disease progression remains to be determined, although the structural differences between the isoforms, which could lead to distinct substrate recognition, should probably account for part of it. HIPK2, an apoptotic inducer, is maintained inactive in unstressed cells through ubiquitination and degradation, which is facilitated by the ubiquitin ligases WSB1 and Siah1 (15). The missing six WD repeats and the SOCS box, which are sufficient for the recognition of HIPK2 in WSB1 ${ }^{3}$ could lead to differential processing of the HIPK2 and, through this mechanism, play a role in determining the fate of NB cells. The significant effect of modulation of the WSB1 isoform expression level was also demonstrated in WSB1-silenced NB cells. Our hypothesis was that if $\mathrm{WSB}^{3}$ was overexpressed in the mice xenografts, then silencing of WSB $1^{3}$ will result in less number of tumorigenic cells. Although siRNAs that target each isoform specifically were insufficient to result in different measurable biological behavior of NB cells, the global effect of WSB1 silencing on viability, apoptosis, and chemotherapy resistance demonstrated in these cells supported our main hypothesis. When all isoforms were targeted by siRNA, NB cells were less viable due to an increased apoptosis rate and enhanced sensitivity to chemotherapeutic agents, suggesting a significant role of WSB1 isoforms in NB biology. Whereas silencing of
WSB1 $1^{1+2}$ alone had no effect on the cells, the expression level of WSB1 $1^{3}$ seemed to play an important role in cell viability. In summary, our data highlight a dominant isoform of the WSB1 gene in several NB systems and suggest a possible role of this isoform in NB cell viability.

\section{METHODS \\ Reagents}

Unless otherwise stated, biochemical reagents were purchased from Sigma Chemical (St Louis, MI).

\section{Cell Lines and Cell Culture Conditions}

The human NB cell lines SK-N-SH, SH-SY5Y, Nub6, IMR32, and SH-EP were cultivated as recommended by American Type Culture Collection (Manassas, VA).

\section{Patients}

Primary tumors were obtained from six MNA NB patients diagnosed and treated at the Pediatric Hematology Oncology Department, Schneider Children's Medical Center of Israel. The local and national Ethics Committees approved the research project. Obtaining informed consent for this study was specifically waived by the Institutional Review Board. All patients were treated according to local protocols that followed published protocols and included vincristine, cisplatin, etoposide, cyclophosphamide, adriamycin, and carboplatin. Median age at diagnosis was 23.3 mo (range: $14-32 \mathrm{mo}$ ). Four out of the six $(66.6 \%)$ patients relapsed. All tissue samples were snap-frozen in liquid nitrogen immediately after surgery and stored at $-80^{\circ} \mathrm{C}$ until use.

\section{Xenograft Tumor Induction in NOD-SCID Mice}

Suspensions of Nub6 cell line, $\left(10^{7} / 100 \mu \mathrm{l}\right.$ PBS $)$ were injected subcutaneously to male and female 6- to 8-week-old NOD-SCID mice (The Jackson Laboratory, Bar Harbor, ME), and tumors were allowed to develop for 14 days.

\section{RNA Isolation and CDNA Preparation}

Purification of mRNA from tissues and cell lines was done by RNAeasy Mini Kit (QIAGEN, Valencia, CA) according to the manufacturer's protocol. cDNA was synthesized using $500 \mathrm{ng}$ RNA by QuantiTect Reverse Transcription Kit (QIAGEN).

\section{RQ-PCR Analysis}

RQ-PCR assays were performed in duplicates and included no-template controls on the Roche LightCycler 480 Probes master system (Roche Diagnostics, Indianapolis, IN). Aliquots $(3 \mu \mathrm{l})$ of cDNA diluted 1:10 were amplified in $10 \mu \mathrm{l}$ of mixture containing $1 \times$ LightCycler 480 Probes Master, $10 \mathrm{pmol}$ primers, and $100 \mathrm{nmol} / \mathrm{l}$ universal ProbeLibrary probe (Roche). Primers used to amplify WSB1 ${ }^{1+2}$ (NM_015626, NM_134265 respectively) were 5'-TCTCCTGACTCTTCTATGCTGTGT-3' and $5^{\prime}$-CATGGTGTATTTATCCATATTCCAAA-3' and universal ProbeLibrary probe \#36. Primers used to amplify WSB1 ${ }^{3}$ (NM_134264) specifically were $5^{\prime}$-GGTTTAACATCCTTGTTGTTTGC-3' and 5'-CCAACTTGAGCCTAGGAAGAGA-3' and universalProbeLibrary probe \#76. The glyceraldehyde-3-phosphate dehydrogenase (GAPDH) RNA was amplified by the 5'-AGCCACATCGCTCAGACAC-3' and $5^{\prime}$-GCCCAATACGACCAAATCC-3' primers and the universal ProbeLibrary probe \#60 and was used as a reference gene. Assays were performed with the following cycle parameters: $10 \mathrm{~min}$ at $95^{\circ} \mathrm{C}$, followed by 55 cycles each consisting of $10 \mathrm{~s}$ at $95^{\circ} \mathrm{C}$ and $30 \mathrm{~s}$ at $60^{\circ} \mathrm{C}$, and a cooling step of $1 \mathrm{~min}$ at $40^{\circ} \mathrm{C}$. To establish a standard curve or relative quantification, cDNAs of the NB tumor pool were tenfold serially diluted and amplified. Quantification was performed against the standard curve, and the individual WSB1 isoforms expression values were subsequently normalized against the matching GAPDH value. Each sample was analyzed in duplicate, and the experiment was repeated at least twice. Results were analyzed using Exor4 (Roche) and expressed as a ratio of GAPDH.

\section{siRNA-Mediated Silencing of WSB1 Expression}

WSB1 expression was silenced in cultured cells with a specific siRNA. Cells were transfected using INTERFERin transfection reagent 


\section{Articles $\mid$ Shichrur et al.}

(Polyplus-transfection, NY) following the manufacturer's instructions with a siRNA1 (sense 5'-GAAAACUCCUCCUUAACUUd(TT)-3'; Sigma) or siRNA2 (ON-TARGET plus SMART pool L-01301500-0005, Human WSB1, NM_134265; Dharmacon, Denver, CO) designed for all three WSB1 isoforms.

\section{XTT/Cell Viability Assays}

NB cells were cultivated $24 \mathrm{~h}$ after siRNA transfection in a flat 96-well plate. To each well, $100 \mu \mathrm{l}$ of growth medium was added, and the cells were left to grow for $24 \mathrm{~h}$. The XTT assay (Biological Industries, Kibbutz Beit Haemek, Israel) was conducted according to the manufacturer's protocol, and the plate was incubated in an incubator for $4 \mathrm{~h}$. XTT metabolism (at an optical density of $450-500 \mathrm{~nm}\left[\mathrm{OD}_{450}-\right.$ $\left.\mathrm{OD}_{500}\right]$ ) was read and normalized to cell density $\left(\mathrm{OD}_{690}\right)$. Cell viability after treatment with doxorubicin for $24 \mathrm{~h}$ or cisplatin for $48 \mathrm{~h}$ was determined by XTT assay in the above protocol.

\section{Apoptosis Analysis}

NB cells were collected in growth media $48 \mathrm{~h}$ after siRNA transfection. Cells were washed with PBS, fixed in cold $70 \%$ ethanol for $24 \mathrm{~h}$ at $-20^{\circ} \mathrm{C}$, and treated using detergent-trypsin mixture (Sigma) method, which was followed by staining with propidium iodide (Sigma) (17) and immediate analysis by flow cytometry (FACSCalibur, Becton Dickinson, Le Pont-De-Claix, France). DNA content and apoptosis were analyzed using the ModFitLT analysis software (Verity Software House, Topsham, ME).

\section{Statistical Analyses}

Statistical analysis was performed using SPSS 18.0 software (SPSS, Chicago, IL), and $P<0.05$ was considered statistically significant. Data are presented as the mean \pm SD.

\section{ACKNOWLEDGMENT}

The authors are grateful to Alla Zozovsky for her technical assistance.

\section{STATEMENT OF FINANCIAL SUPPORT}

This work was supported by the Rina Zaizov Memorial Fund.

Disclosure: The authors declare no conflict of interest.

\section{REFERENCES}

1. Smith MA, Seibel NL, Altekruse SF, et al. Outcomes for children and adolescents with cancer: challenges for the twenty-first century. J Clin Oncol 2010;28:2625-34.

2. D’Angio GJ, Evans AE, Koop CE. Special pattern of widespread neuroblastoma with a favourable prognosis. Lancet 1971;1:1046-9.
3. De Bernardi B, Pianca C, Boni L, et al. Disseminated neuroblastoma (stage IV and IV-S) in the first year of life. Outcome related to age and stage. Italian Cooperative Group on Neuroblastoma. Cancer 1992;70:1625-33.

4. Maris JM. Recent advances in neuroblastoma. N Engl J Med 2010;362:220211.

5. Westermark UK, Wilhelm M, Frenzel A, Henriksson MA. The MYCN oncogene and differentiation in neuroblastoma. Semin Cancer Biol 2011;21:256-66

6. Gustafson WC, Weiss WA. Myc proteins as therapeutic targets. Oncogene 2010;29:1249-59.

7. Li MH, Hla T, Ferrer F. Sphingolipid modulation of angiogenic factor expression in neuroblastoma. Cancer Prev Res (Phila) 2011;4:1325-32.

8. Liberman J, Sartelet H, Flahaut M, et al. Involvement of the CXCR7/ CXCR4/CXCL12 axis in the malignant progression of human neuroblastoma. PLoS ONE 2012;7:e43665.

9. Turner KE, Kumar HR, Hoelz DJ, et al. Proteomic analysis of neuroblastoma microenvironment: effect of the host-tumor interaction on disease progression. J Surg Res 2009;156:116-22.

10. Archange C, Nowak J, Garcia S, et al. The WSB1 gene is involved in pancreatic cancer progression. PLoS ONE 2008;3:e2475.

11. Chen QR, Bilke S, Wei JS, et al. Increased WSB1 copy number correlates with its over-expression which associates with increased survival in neuroblastoma. Genes Chromosomes Cancer 2006;45:856-62.

12. David CJ, Manley JL. Alternative pre-mRNA splicing regulation in cancer: pathways and programs unhinged. Genes Dev 2010;24:2343-64.

13. Dentice M, Bandyopadhyay A, Gereben B, et al. The Hedgehog-inducible ubiquitin ligase subunit WSB-1 modulates thyroid hormone activation and PTHrP secretion in the developing growth plate. Nat Cell Biol 2005;7:698 705.

14. Vasiliauskas D, Hancock S, Stern CD. SWiP-1: novel SOCS box containing WD-protein regulated by signalling centres and by Shh during development. Mech Dev 1999;82:79-94.

15. Choi DW, Seo YM, Kim EA, et al. Ubiquitination and degradation of homeodomain-interacting protein kinase 2 by WD40 repeat/SOCS box protein WSB-1. J Biol Chem 2008;283:4682-9.

16. Zeöld A, Pormüller L, Dentice M, et al. Metabolic instability of type 2 deiodinase is transferable to stable proteins independently of subcellular localization. J Biol Chem 2006;281:31538-43.

17. Vindeløv LL, Christensen IJ, Nissen NI. A detergent-trypsin method for the preparation of nuclei for flow cytometric DNA analysis. Cytometry 1983;3:323-7. 